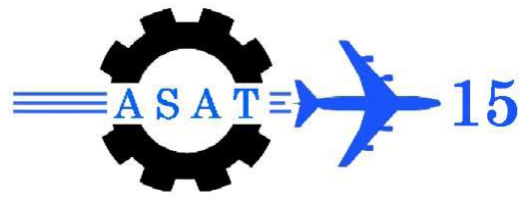

\title{
Enhancement the Spectral Efficiency of Half-Duplex Single Antenna Multi-Relay System
}

\author{
Ahmed El-Mahdy ${ }^{*}$, Ahmed Afifi $^{\dagger}$, and Nourhan Nabil ${ }^{\star}$
}

\begin{abstract}
In this paper, we study two-hop communication protocols where several relay terminals assist in the communication between two terminals. The terminals operate in halfduplex mode, hence the transmission of one information symbol from the source terminal to the destination terminal occupies two channel uses. This leads to a loss in spectral efficiency due to the per-log factor one-half in corresponding capacity expressions. In this paper, we propose to apply two-path and two-way protocols to multi-relay networks. These relay protocols avoid the pre-log factor one-half. The communication channels between source and relays and between the relays and destination are assumed to be Rayleigh flat fading. The performance of the network is investigated for perfect and estimated channel and for decodeand-forward scheme. The performance is measured in terms of bit error rate. Moreover the outage probability is evaluated when one relay is used and is compared with that one for multi-relay system. Channel estimation is performed using least square algorithm. The performance of the channel estimation algorithm is measured in terms of the mean square error of estimation.
\end{abstract}

Keywords: Wireless communication, relay channel, half-duplex, decode and forward, fading channel.

\section{Introduction}

Cooperative relaying as a promising technology has attracted widespread attentions, since it can achieve higher transmission quality and throughput for wireless networks [1]. The main idea is to allow several neighboring nodes help one other by cooperating among themselves [2]. In cooperative communication, the relay nodes can help the source to improve the performance at the destination [3]. It fundamentally changes the abstraction of a wireless link and offer significant potential advantages for wireless communication networks [4]. In [4] the authors address a two-path relaying protocol assuming a unidirectional traffic model using two half-duplex relays that assist in the communication assuming that there is no direct connection between terminals source and destination is available and Transmission of messages from a source to a destination is done via two relays which do not receive and transmit simultaneously also addressing a two-way relaying protocol assuming a bidirectional traffic model in order to compensate the spectral efficiency loss. In [5] the authors address two IRI cancellation schemes that exploit the orthogonal projection methods using multiple antennas at the relay nodes. In [6] the authors analyze the fundamental performance of twopath in terms of the diversity-multiplexing tradeoff (DMT) by deriving the DMT for the

Professor in the faculty of information Engineering \& Technology (IET), German University in Cairo. E-mail: ahmedelmahdy@guc.edu.eg .

$\dagger \quad 4^{\text {th }}$ year student in the faculty of IET. ahmed.El-nemer@student.guc.edu.eg

$\ddagger \quad 4^{\text {th }}$ year student in the faculty of IET. nourhan.el-sharnoby@student.guc.edu.eg 
protocol where perfect decoding at the relays is assumed, and shown that it approaches the $3 \times 1$ multiple-input single-output (MISO) DMT and then removing the assumption of perfect decoding at relays and derive the closed-form expression of the achievable DMT based on the relative distances nodes. In [7] and [8], the authors address conventional scheme, less transmission cycles are required in two-way relaying, which consequently provides the improvement in performance of spectral efficiency. Recently, two-way DF relaying has been studied in many points of views. As an important performance measure, outage probability has been investigated for two-way communication in the literature. Meanwhile, papers [9], [10] and [11] conducted the outage analysis for the DF scheme. However, in [9] the two individual outage events are still separately examined. In [10] and [11], the authors consider symmetric traffics in their analysis.

In this paper, we extend the two way and two path protocols to the case of multi relay systems to mitigate the loss in the spectral efficiency due to half-duplex operation of the relays. We evaluate the performance of two-path and two-way relaying systems in perfect and estimated channels. The relays are assumed to be operated with decode and forward scheme.

\section{System Model}

We consider a two-hop network model where there is one source, one destination and $\mathrm{K}$ relays as shown in Fig. 1. We assume that there is no direct communication between the source and the destination. The source, relays, and the destination are deployed with single antenna. The total number of relays is $\mathrm{n}$. we limit our discussion to the case where the channels are flat fading. The transmission of the data is done in two time slots. The network uses decode and forward protocol. In this protocol, the source encodes the message and transmits it to the relays, then the relays decode the message, re-encode it by the same or a different codebook and forward the message to the destination then the destination decodes the received message and extracts the original data from it.

\section{Two-Path and Two-Way Decode and Forward for Multi-Relay System}

In order to increase the spectral efficiency and the signal strength, we propose multi-relay system using two-path and two-way protocols. By combining the received signals send from the relays, cooperative diversity can be achieved. It has been shown that cooperative communications can dramatically improve the system capacity and performance. We are assuming that all terminals are operating in half-duplex mode, the relays are using decode and forward scheme and there is no direct connection between the source terminal and the destination terminal is available.

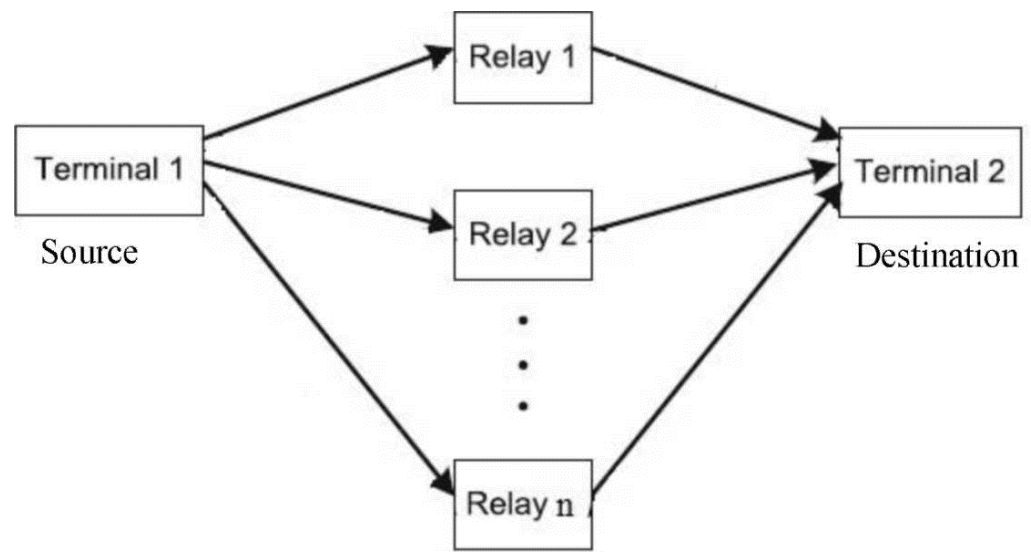

Fig. 1 Multi-relay communication system 


\section{A. Two-Path Relaying}

In two-path relaying scheme, the message is sent in two time slots. In the first time slot the source transmits the message to the $\mathrm{n}$ relays and in the second time slot the message is forwarded to the destination as shown in Fig. 1 . Where $R_{n}$ should always be an even number as in two-path method the transmission is done via two relays. The length of time slot is equal to the length of one code word (frame) and is NT, where T is the sampling interval and $\mathrm{N}$ is the number of symbols in each frame. In odd time slots, $\mathrm{k}=1,3,5, \ldots$, half of the relays will be receiving whereas the other half would be transmitting (except for $\mathrm{k}=1$ where the relays should transmit do not transmit). In even time slots, $\mathrm{k}=2,4,6, \ldots$, the relays were receiving in the odd time slot will be transmitting and the ones were transmitting will be receiving. A system with 8 relays is given in Table.1. This cooperation protocol avoids the pre-log factor one-half since the source transmits a new message in every time slot and has not to be silent in each second time slot. The receiver is using maximal ratio combining (MRC) to combine the messages coming from half of the relays every time slot and extract the information. The equations that describe this scheme is as follows. The received signal at the relays is given by

$$
y_{i}[k]=h_{i}[k] x_{1}[k]+n_{i}[k]
$$

where $h_{i}[k]$ is the complex channel gain from source to relay $T_{i}, x_{l}[k]$ is the transmitted symbol of the source, and $n_{i}[k]$ is additive white Gaussian noise at the relay $T_{i}$.

$$
h_{i}[k]=A_{i} e^{j \varphi_{i}}
$$

where $A_{i}$ is the random magnitude of the $i^{\text {th }}$ channel which has a Rayleigh distribution and $\varphi_{i}$ is the random phase of $i^{\text {th }}$ channel which has a uniform distribution from $[0,2 \pi]$.

After the message is decoded, re-encoded and transmitted to the destination, the received signal from each relay is given by

$$
y_{2}[k+1]=h_{i}[k+1] x_{i}[k+1]+n_{2}[k+1]
$$

where, $h_{i}[k+1]$ is the complex channel gain from relay $T_{i}$ to the destination, $x_{i}[k+1]$ is the transmitted symbol of the relay $T_{i}$, and $n_{2}[k+1]$ is additive white Gaussian noise at the destination. At the receiver first, the receiver divide what it has received from each relay by $h_{i}[k+1]$ and then combing them together (MRC) and extracting the information from it.

\section{B. Two-Way Relaying}

In the Two way Relaying Protocol the overall transmission is done in two time slots. In the first time slot the source and the destination send their respective information symbols to all $\mathrm{n}$ Relays. Let $b_{s}[k]$ and $b_{d}[k]$ represent the $k^{\text {th }}$ information bit transmitted by the source and destination and $x_{s}[k]$ and $x_{d}[k]$ denote the corresponding modulated symbols, respectively.

Table 1. Two-path relaying algorithm with 8 relays

\begin{tabular}{c|ccccccc}
\hline \hline$T$ & $\mathrm{Tx}$ & $\mathrm{Tx}$ & $\mathrm{Tx}$ & $\mathrm{Tx}$ & $\mathrm{Tx}$ & $\mathrm{Tx}$ & $\mathrm{Tx}$ \\
\hline$T_{1}$ & $\mathrm{Rx}$ & $\mathrm{Tx}$ & $\mathrm{Rx}$ & $\mathrm{Tx}$ & $\mathrm{Rx}$ & $\mathrm{Tx}$ & $\mathrm{Rx}$ \\
\hline$T_{2}$ & $\mathrm{Rx}$ & $\mathrm{Tx}$ & $\mathrm{Rx}$ & $\mathrm{Tx}$ & $\mathrm{Rx}$ & $\mathrm{Tx}$ & $\mathrm{Rx}$ \\
\hline$T_{3}$ & $\mathrm{Rx}$ & $\mathrm{Tx}$ & $\mathrm{Rx}$ & $\mathrm{Tx}$ & $\mathrm{Rx}$ & $\mathrm{Tx}$ & $\mathrm{Rx}$ \\
\hline$T_{4}$ & $\mathrm{Rx}$ & $\mathrm{Tx}$ & $\mathrm{Rx}$ & $\mathrm{Tx}$ & $\mathrm{Rx}$ & $\mathrm{Tx}$ & $\mathrm{Rx}$ \\
\hline$T_{5}$ & & $\mathrm{Rx}$ & $\mathrm{Tx}$ & $\mathrm{Rx}$ & $\mathrm{Tx}$ & $\mathrm{Rx}$ & $\mathrm{Tx}$ \\
\hline$T_{6}$ & & $\mathrm{Rx}$ & $\mathrm{Tx}$ & $\mathrm{Rx}$ & $\mathrm{Tx}$ & $\mathrm{Rx}$ & $\mathrm{Tx}$ \\
\hline$T_{7}$ & & $\mathrm{Rx}$ & $\mathrm{Tx}$ & $\mathrm{Rx}$ & $\mathrm{Tx}$ & $\mathrm{Rx}$ & $\mathrm{Tx}$ \\
\hline$T_{8}$ & & $\mathrm{Rx}$ & $\mathrm{Tx}$ & $\mathrm{Rx}$ & $\mathrm{Tx}$ & $\mathrm{Rx}$ & $\mathrm{Tx}$ \\
\hline$R$ & & $\mathrm{Rx}$ & $\mathrm{Rx}$ & $\mathrm{Rx}$ & $\mathrm{Rx}$ & $\mathrm{Rx}$ & $\mathrm{Rx}$ \\
\hline \hline
\end{tabular}


Then the corresponding received signal at the $i^{\text {th }}$ relay transmitted from the source denoted by $y_{i s}[k]$ can be expressed as

$$
y_{i s}[k]=h_{i s}[k] x_{s}[k]+n_{i s}[k]
$$

where $h_{i s}[k]$ denotes the fading coefficient between the source and the $i^{\text {th }}$ relay and $n_{i s}[k]$ is the additive white Gaussian noise in the channel between the source and the relay. Consequently, the corresponding received signal at the $i^{\text {th }}$ relay transmitted from the destination can be expressed as

$$
y_{i d}[k]=h_{i d}[k] x_{d}[k]+n_{i d}[k]
$$

where $h_{i d}[k]$ is the fading coefficient between the destination and the $i^{\text {th }}$ relay and $n_{i d}[k]$ is the additive white Gaussian noise in the channel between the destination and the relay.

The relays decode the received signals from the source and the destination and perform the network coding. Then, in the second time slot the relays broadcast the signal to both the source and destination. The received signal from the $i^{\text {th }}$ relay at the source and destination is given by

$$
y_{i r}[k+1]=h_{i s}[k+1] x_{s}[k+1]+h_{i d}[k+1] x_{d}[k+1]+n_{i s}[k+1]+n_{i d}[k+1]
$$

Then, the signals received from all relays are combined at the source and destination using MRC (assuming the channel is known by estimation). The source and destination decode the received signal and estimates

$$
b_{r}[k]=b_{s}[k] \oplus b_{d}[k]
$$

Let $\tilde{b}_{s, r}[k]$ and $\tilde{b}_{d, r}[k]$ represent the estimated $b_{r}[k]$ at the source and the destination respectively. Since the source knows its own transmitted bits $b_{s}[k]$, it can recover the destination bits by simply XORing $\tilde{b}_{d, r}[k]$ with its own bits $b_{d}[k]$ denoted by $\tilde{b}_{d i}[k]$

$$
\tilde{b}_{d i}[k]=\tilde{b}_{s, r}[k] \oplus b_{s}[k]
$$

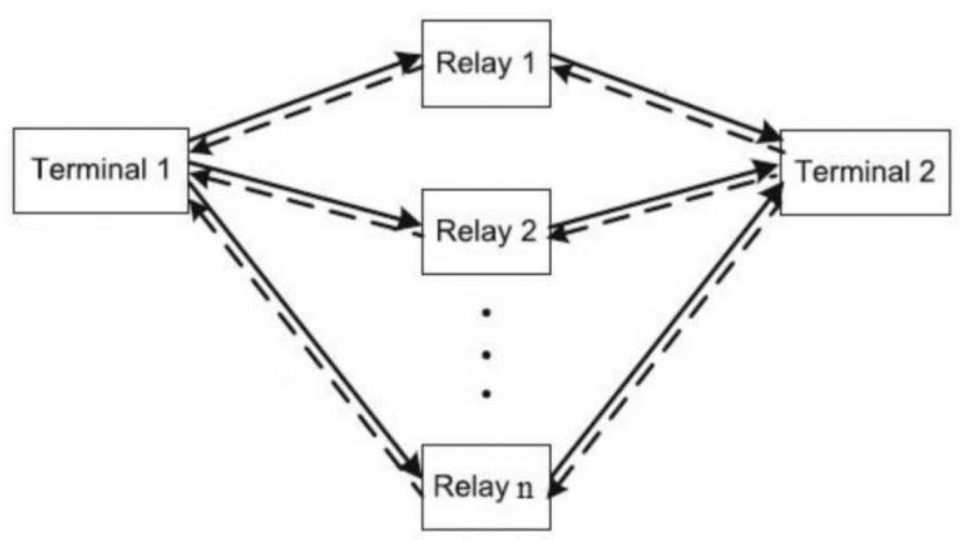

Fig. 2 Multi-relay using two-way protocol 


\section{Channel Estimation}

Channel estimators are used to obtain the unknown fading channel complex gain. Each potential relay estimates the instantaneous channel quality between itself and the source (S) as well as itself and destination (D), respectively. This could be achieved as follows: Node $S$ sends a ready-to-send (RTS) message which is received by the $\mathrm{N}$ relays. Upon the reception of RTS, the N relays send back a clear-to-send (CTS) message. Both messages are pilot signals. They contain no further information and are transmitted with the same carrier frequency and the same power used for the payload data transmission later on. By receiving the RTS message the $\mathrm{N}$ relays can determine the channel state information from the source to each of them. Then the N relays send RTS message which is received by the destination and upon the reception of RTS. The destination sends back a CTS message to the $\mathrm{N}$ relays. Also both messages are pilot signals. By receiving the RTS, the destination can determine the channel state information from each relay. The received signal at the relays is given by:

$$
r_{i}(t)=h_{i} s_{Q}(t)+n
$$

where $h_{i}$ is the required channel complex gain and $S_{Q}(t)$ is the pilot signal. By using least square method the channel complex gain is estimated as follows:

$$
\widehat{h_{l}}=\left(\boldsymbol{S}_{Q}{ }^{T} \boldsymbol{S}_{Q}\right)^{-1} \boldsymbol{S}_{Q} \boldsymbol{r}_{i}
$$

Where $S_{Q}$ is the pilot signal vector containing the pilot sequence of bits and $\boldsymbol{r}_{i}$ is the received vector. The performance of the least square algorithm is measured in terms of mean square error (MSE) which is one of many ways used to measure the accuracy of the estimation algorithm. Mean square error defines the significance of the channel estimator and it is given by

$$
M S E=E\left(\left|\tilde{h}_{i}-h_{i}\right|^{2}\right)
$$

where $\mathrm{E}($.$) denotes the expectation.$

\section{Simulation and Results}

In this section, numerical results are represented to evaluate the performance of the multirelay networks when two-path and two-way protocols are used. The results are obtained for perfect and estimated channels. The simulation parameters are as follows. The number of bits is 100,000; the type of modulation is binary phase shift key (BPSK), the number of pilot bits used for channel estimation is 100 bits. In two-path protocol, 8 relays are used and in twopath protocol 4 relays are used. MRC is used to combine the signals.

The BER of the network using the two path and two way protocols for the perfect and estimated channel is shown in Figs. 3 and 4 respectively. The results show that there is a gap between the BER for perfect and estimated channel about $0.5 \mathrm{~dB}$ at $10^{-4} \mathrm{BER}$. This gap is due to the channel estimation errors which affect the BER.

Outage probability is another commonly used performance measure of digital communications. The outage is defined as the probability that the output instantaneous SNR $\gamma$ falls below a certain given threshold $\gamma_{T}$ that is: $P_{\text {out }}=\operatorname{Pr}\left(0 \leq \gamma \leq \gamma_{T}\right)$. Fig. 5 illustrates the outage probability when one relay is used and for $\gamma_{T}=13 \mathrm{~dB}, 16 \mathrm{~dB}$, and $17 \mathrm{~dB}$. Figure 6 illustrates the outage probability for the two-path protocol when 8 relays are used. These figures show that for a fixed SNR as the threshold increases, the outage probability increases. By comparing the two figures we can see that the outage probability when using 8-relays is 
better than that one when using one relay. This is because a MRC is used to combine the signals which have optimum performance.

All the channels between source and the relays and between the relays and destination are estimated using the least square algorithm. We present only second channel from both sides as an example. The absolute of perfect and estimated channel between source and second relay and between second relay and the destination (channel 2) is shown in Figs. 7 and 8 respectively. The results show that at low SNR, the noise dominates the performance and there is a gap between the true and estimated channel. When SNR increases, the estimated channel converges to the true one.

Another measure of channel estimation accuracy is the normalized mean square error of estimation which is plotted in Figs. 9 and 10 for the $n$ channels between the source and the relays and between the $\mathrm{n}$ relays and destination respectively. These figures are obtained by calculating the square error for the $\mathrm{n}$ channels between source and relays and then mean of them is evaluated to obtain NMSE of estimation. The results show that the NMSE of estimation decreases as SNR increases until reach small values after $\mathrm{SNR}=15 \mathrm{~dB}$.

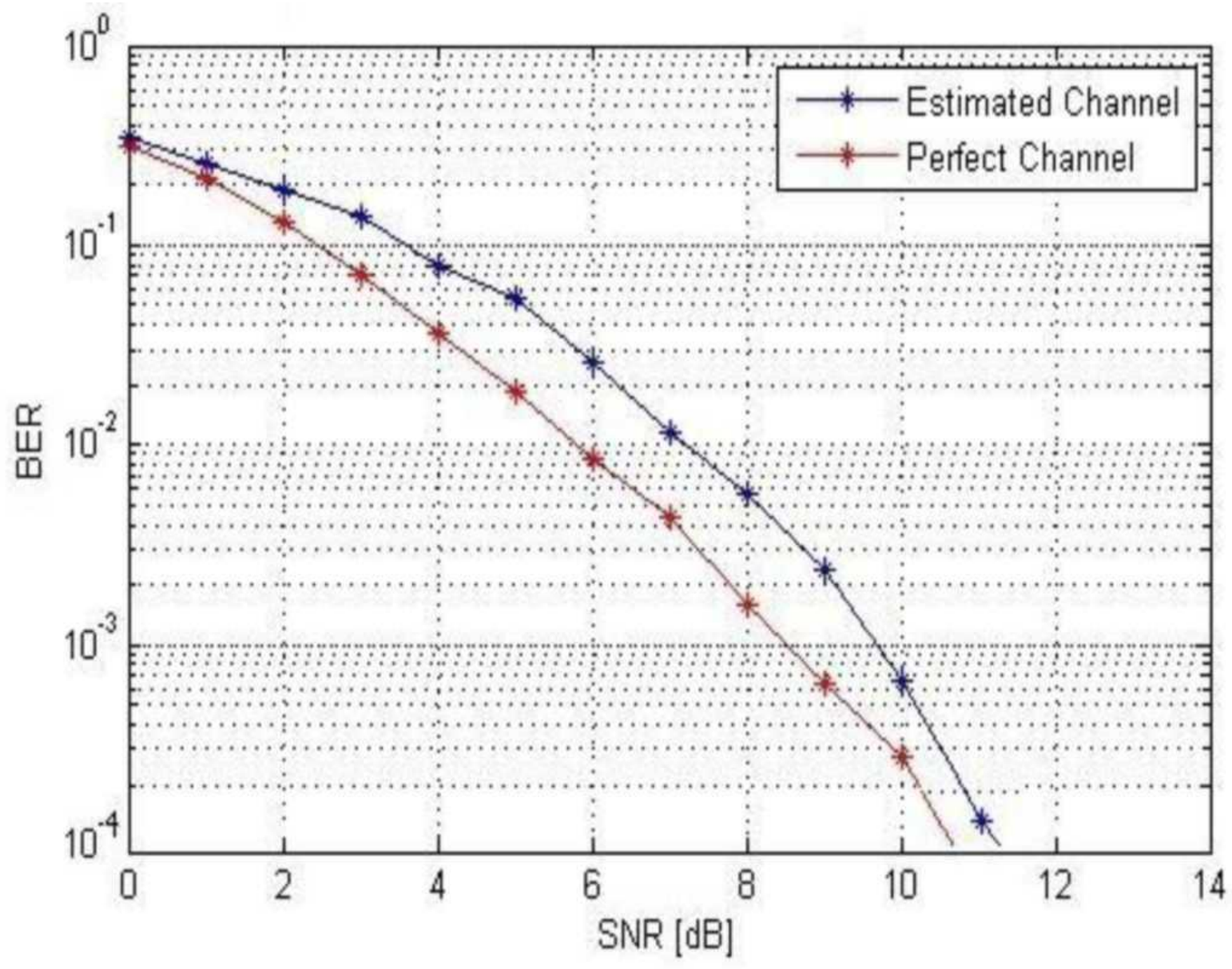

Fig. 3 BER of the network using two-path protocol for the perfect and estimated channels 


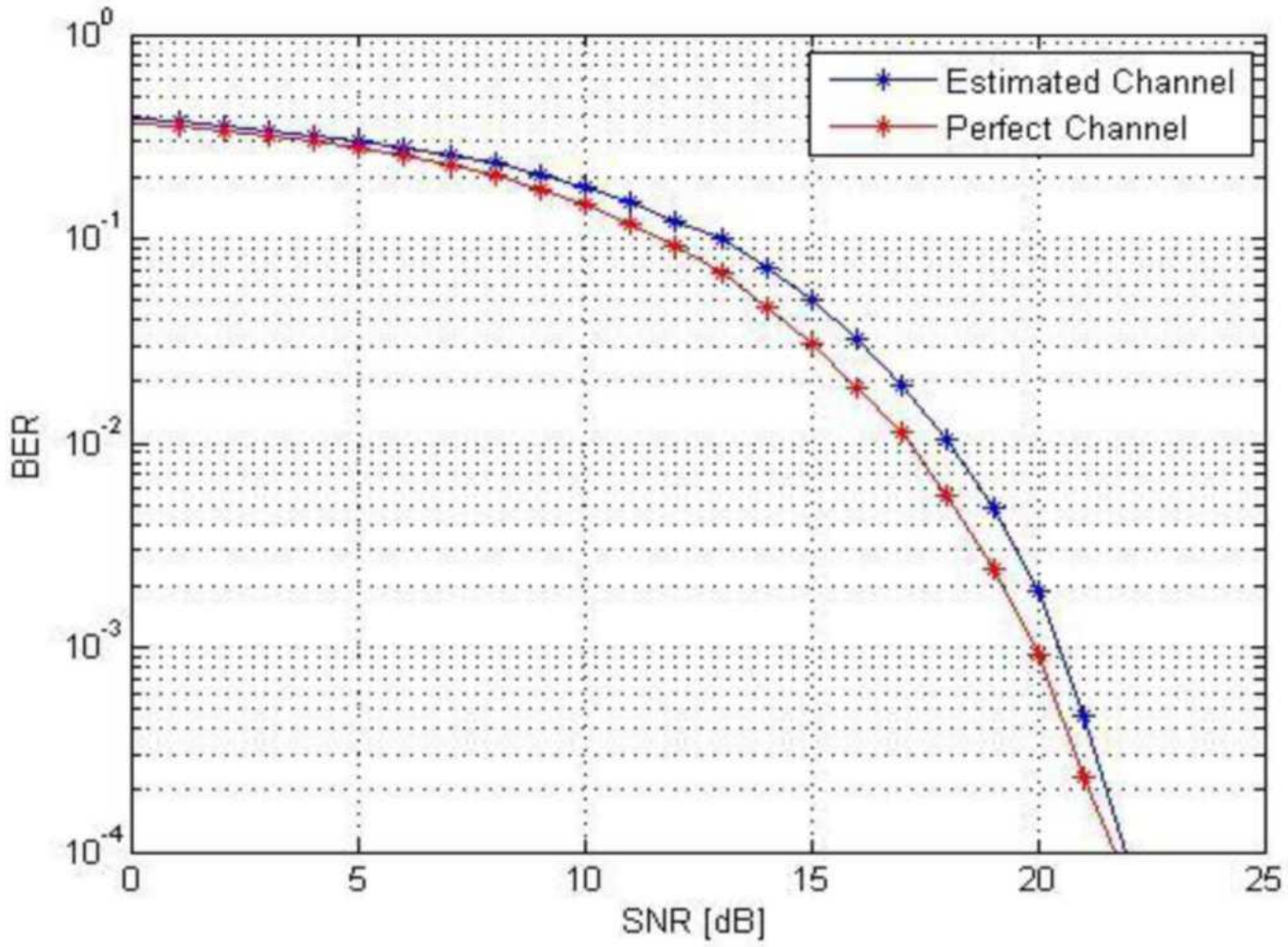

Fig. 4 BER of the network using two-way protocol for the perfect and estimated channels

Outage Probability from source to destination

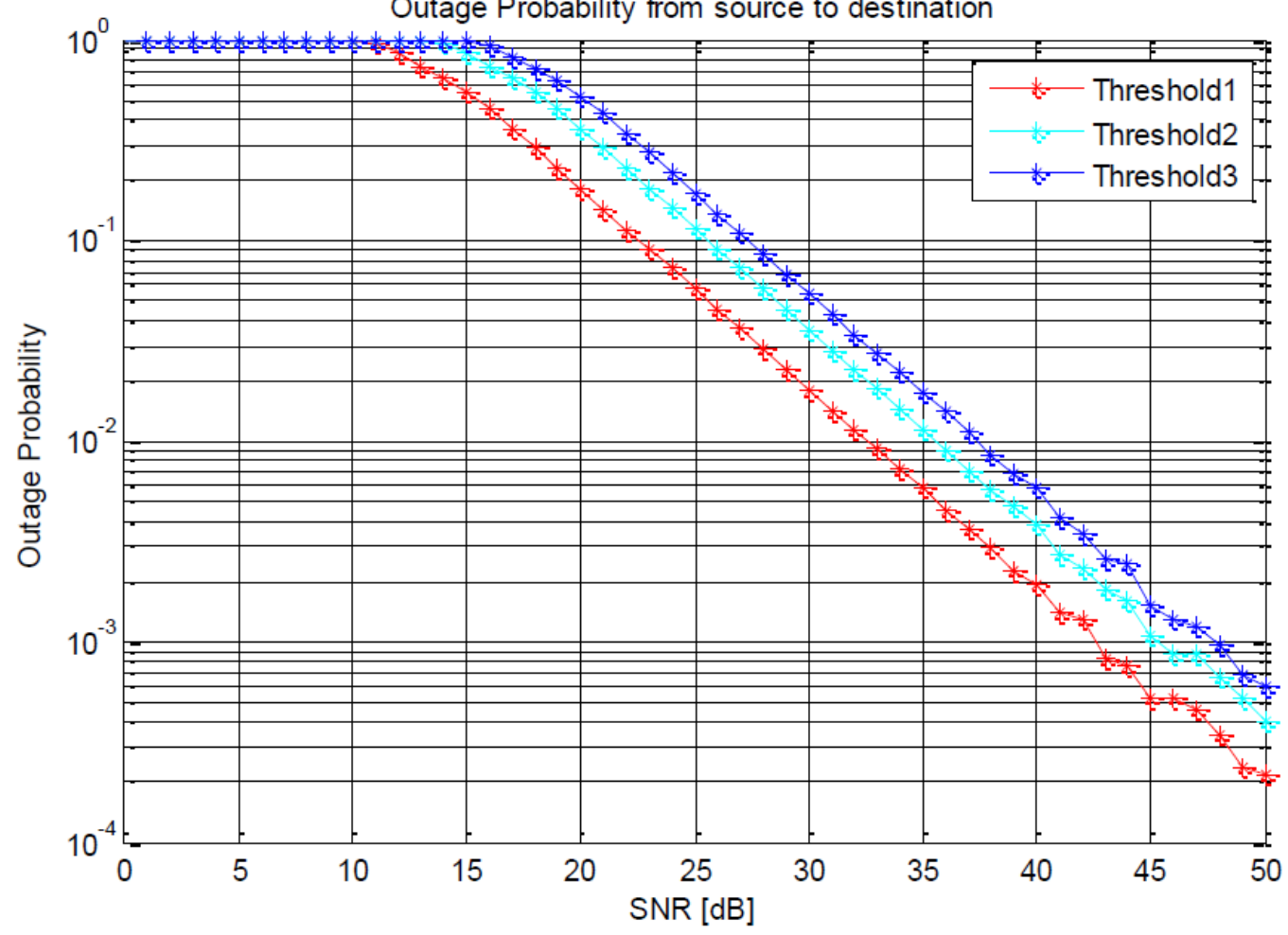

Fig. 5 Outage probabilities for different threshold values and for one relay only 


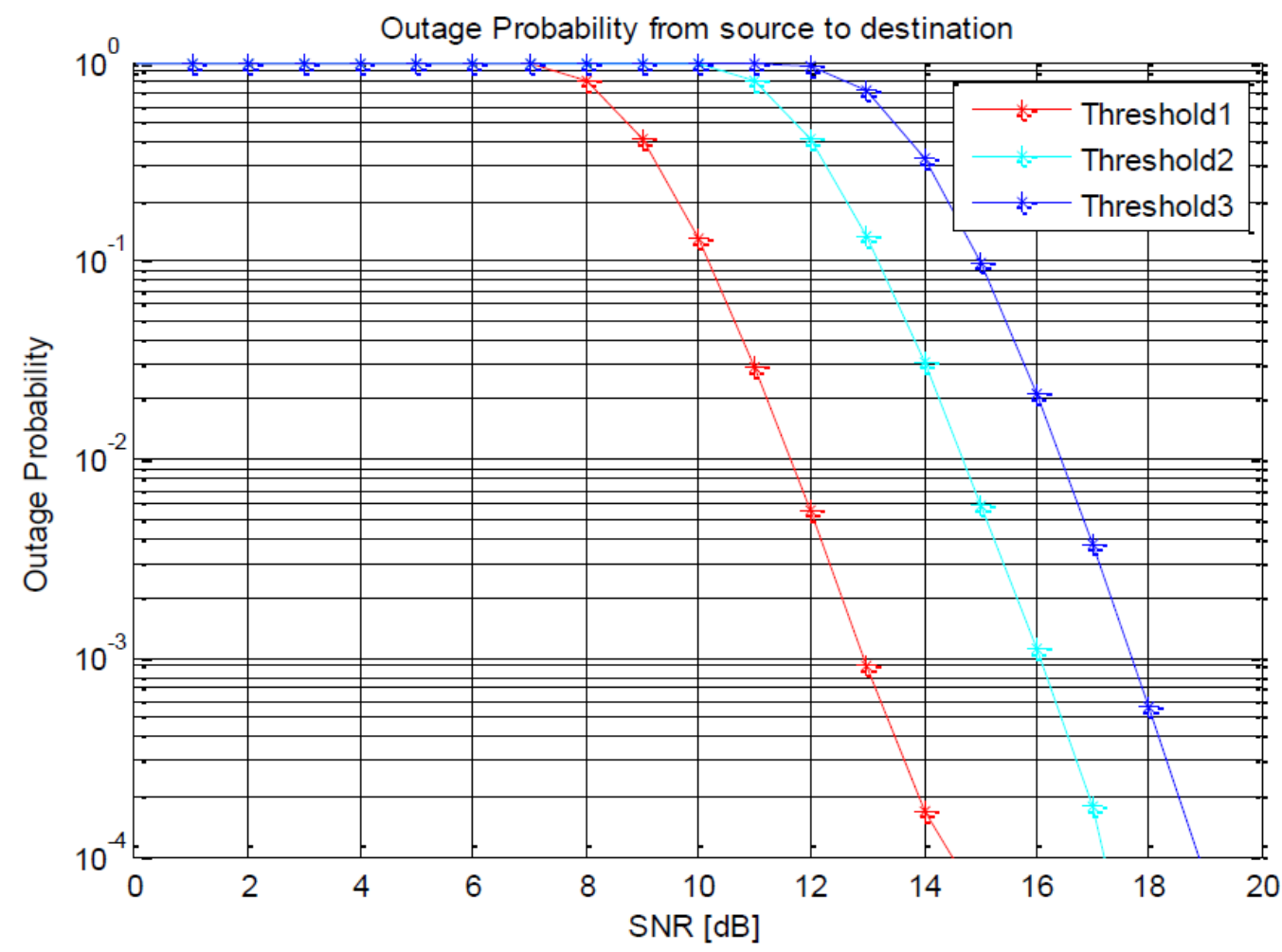

Fig. 6 Outage probabilities of eight relays using the two-path protocol and for different number of thresholds

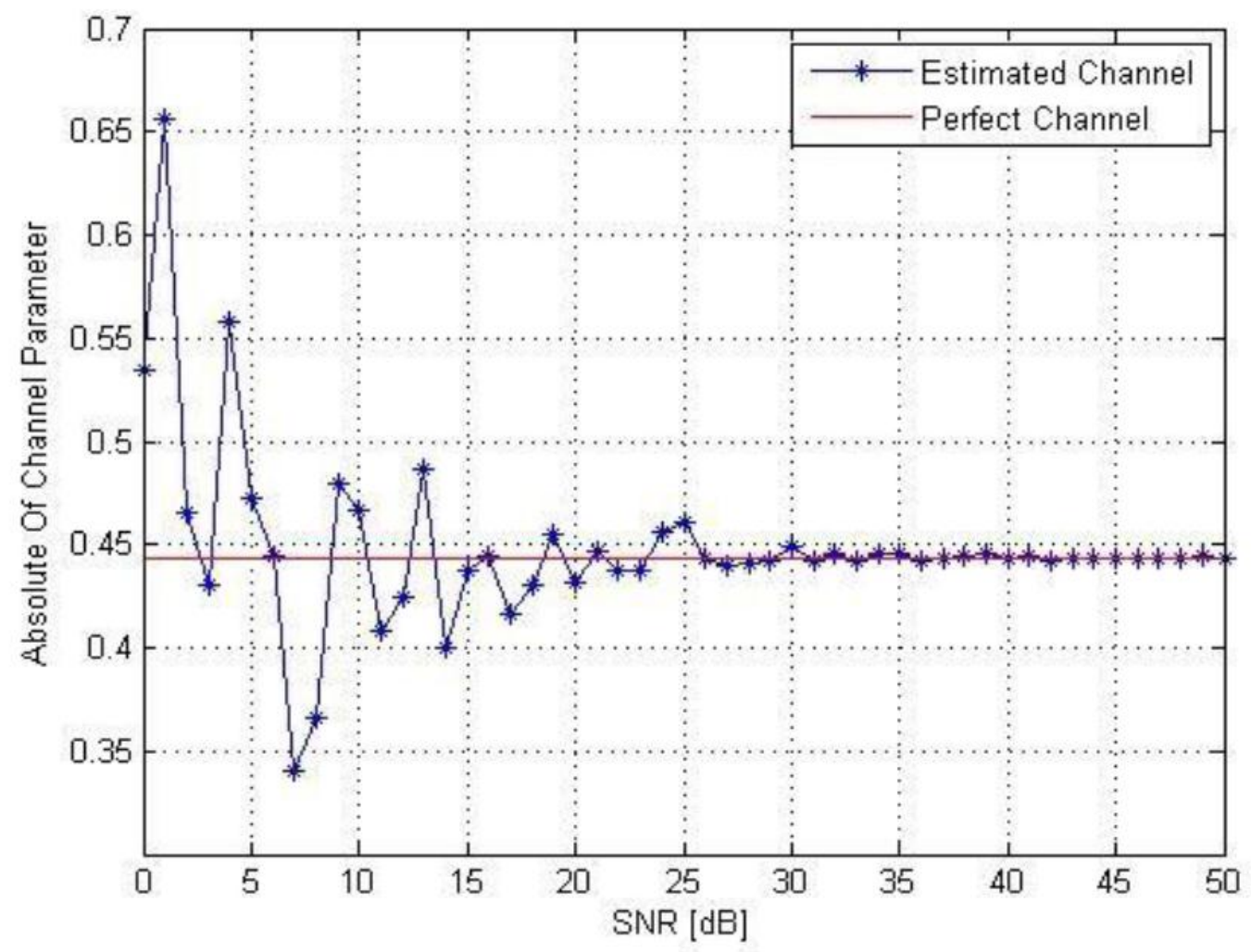

Fig. 7 Absolute values of perfect and estimated channels between source and second relay 


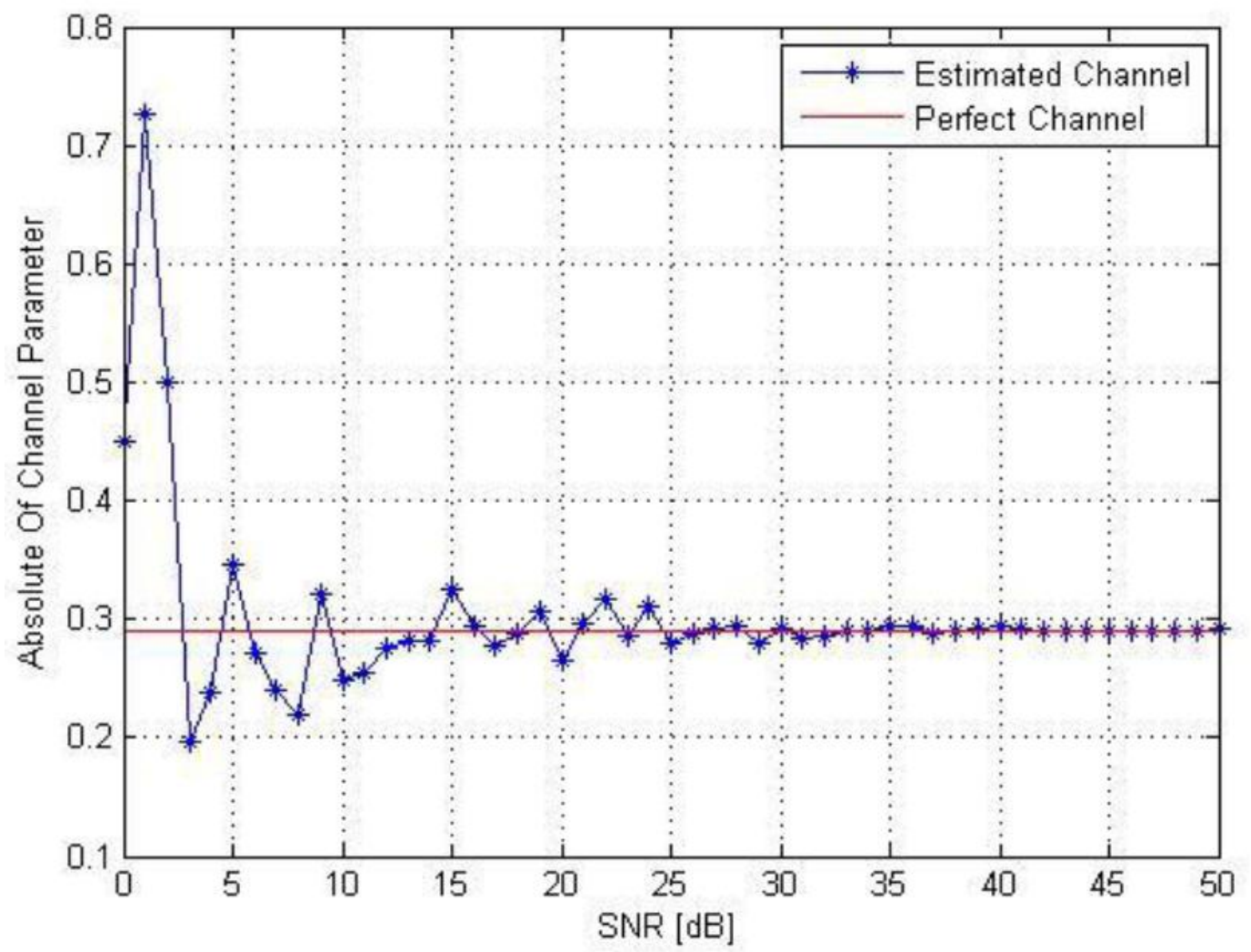

Fig. 8 Absolute values of perfect and estimated channels between second relay and destination

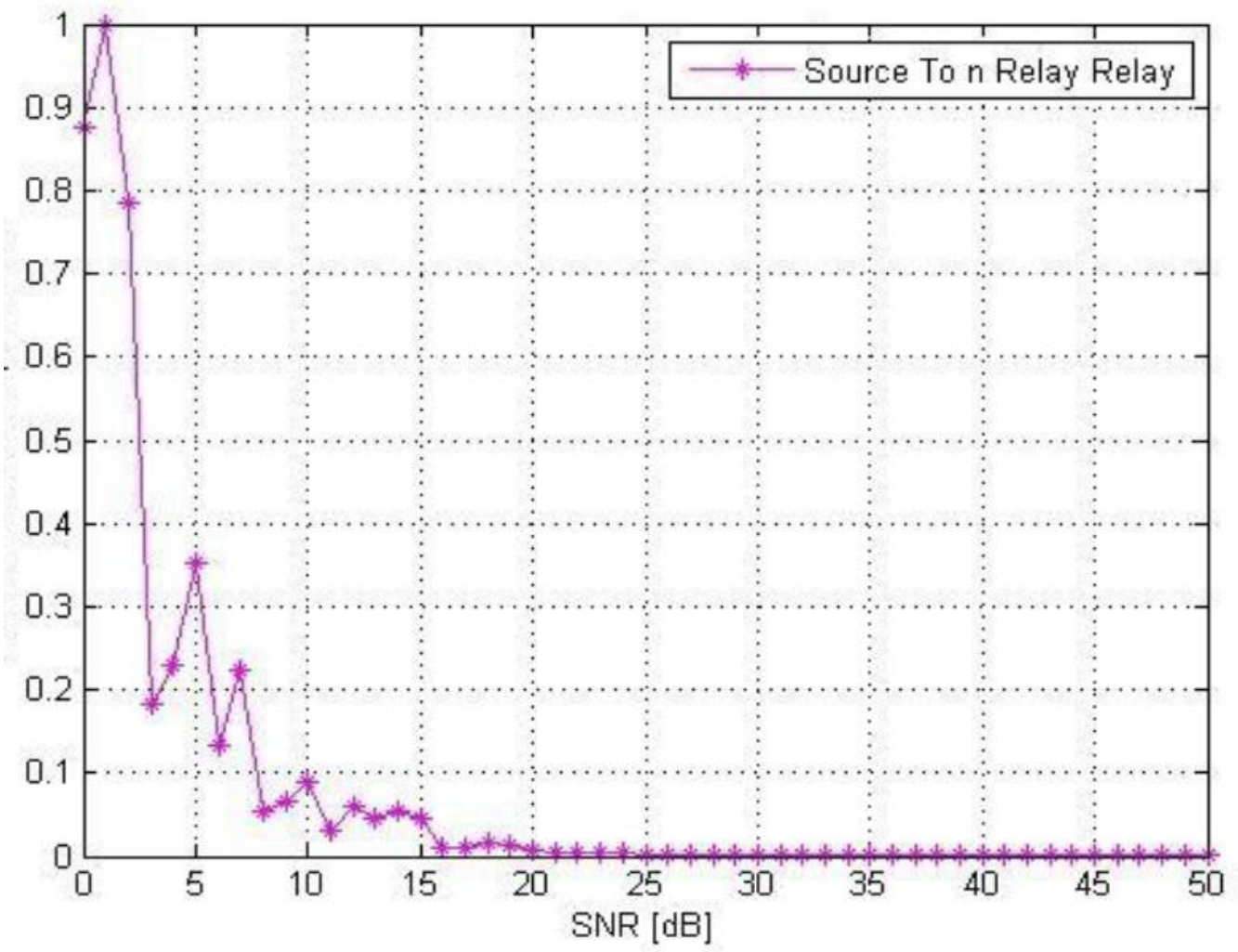

Fig. 9 Normalized mean square error of estimation of the channels between the source and the $n$ relays 


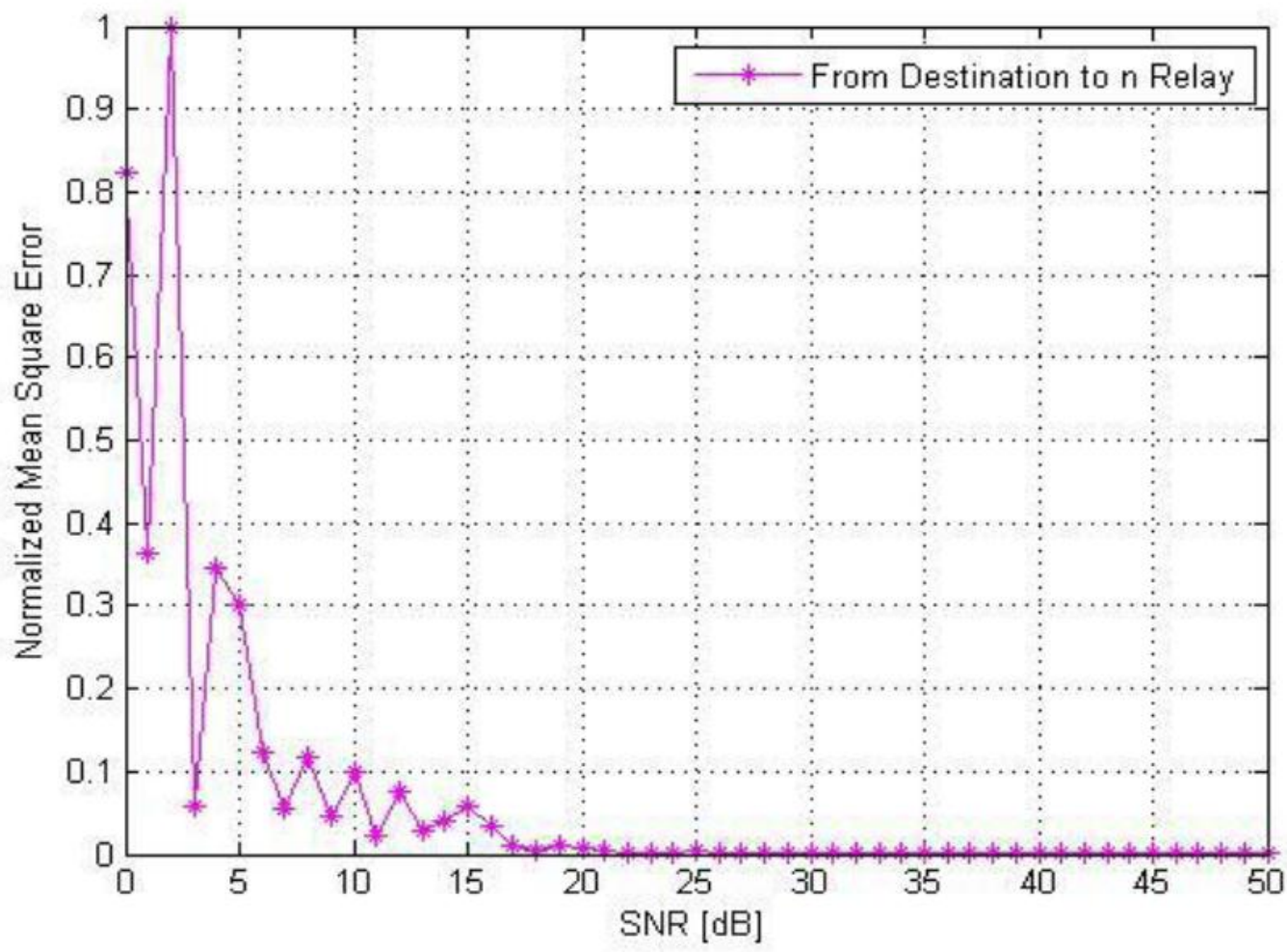

Fig. 10 Normalized mean square error of estimation of the relays and the destination

\section{Conclusions}

In this paper, the two-way and two-path protocols have been applied to multi-relay networks. Decode and forward scheme are used for perfect and estimated channels. These protocols used to enhance the spectral efficiency of the multi-relay networks. The channels between source and relays and between relays and destination have been estimated using least square algorithm. Simulation results show that there is a gap between the BER for perfect and estimated channel about $0.5 \mathrm{~dB}$ at $10-4$ BER due to the estimation error. Moreover, the outage probability is greatly enhanced when using multi-relay system.

\section{References}

[1] Li.Yonghui, H.Y. Raymond and B. Vucetic, "Relay Selection with Network Coding in Two-Way Relay Channels," IEEE Trans. Veh. Tech., vol. 59, pp. 4489-4499, Nov. 2010.

[2] Xu. Feng, F.C.M. Lau, Q.F. Zhou and Dian-Wu Yue. "Outage Performance of Cooperative Communication Systems Using Opportunistic Relaying and Selection Combining Receiver,"IEEE Sig. Proc. Letter., vol. 16. pp. 113-116, Feb. 2009.

[3] C. Min-Kaun and L. Shi-Yong. "Performance Analysis of Cooperative Communication System withHierarchical Modulation over Rayleigh Fading Channel," IEEE Trans. Wireless Commun., vol. 8. pp. 2848-2852, June 2009.

[4] T. Xiaofeng, X. Xiaodong and C.Qimei. "An overview of cooperative communications," IEEE Commun. Mag., vol. 50. pp. 65-71, June 2012.

[5] P. Heesun and C. Joohwan "Inter-Relay Interference Cancellation for AF MFMO TwoPath Relay Systems" in IEEE veh. Tech. Conf., pp. 1-5, Sept 2012. 
[6] H. Wicaksana, S.H. Ting, Y.L. Guan and X.-G. Xia. "Decode-and-Forward Two-Path Half-Duplex Relaying: Diversity-Multiplexing Tradeoff Analysis" IEEE Trans. Commun., vol. 59. pp. 1985-1994, July 2011.

[7] Q. Li, S. H. Ting, A. Pandharipande, and Y. Han, "Adaptive two-way relaying and outage analysis," IEEE Trans. Wirel. Commun., vol. 8, no. 6, pp. 3288-3299, 2009.

[8] I. Krikidis, "Relay selection for two-way relay channels with MABC DF: a diversity perspective," IEEE Trans. Veh. Technol., vol. 59, no. 9, pp. 4620-4628, 2010.

[9] Z. Ding, K. K. Leung, D. L. Goeckel, and D. Towsley, "On the study of network coding with diversity," IEEE Trans. Wirel. Commun., vol. 8, no. 3, pp. 1247-1259, 2009.

[10] P. Liu, and I. M. Kim, "Performance analysis of bidirectional communication protocols based on decode and forward relaying, IEEE Trans. Commun., vol. 58, no. 9, pp. 26832696, 2010.

[11] I. Krikidis, "Relay selection for two-way relay channels with MABC DF: a diversity perspective," IEEE Trans. Veh. Technol., vol. 59, no. 9, pp. 4620-4628, 2010. 